\title{
Deflectometric method for the measurement of user power for ophthalmic lenses
}

\author{
Javier Vargas, ${ }^{1}$ José A. Gómez-Pedrero, ${ }^{2, \star}$ José Alonso, ${ }^{2}$ and Juan A. Quiroga ${ }^{3}$ \\ 'Laboratorio de instrumentación espacial (LINES), Instituto Nacional de Técnica Aerospacial (INTA), \\ Carretera de Ajalvir, km 4, 28860 Torrejón de Ardoz, Madrid, Spain \\ ${ }^{2}$ Optics Department, School of Optics, Universidad Complutense de Madrid, Avda. \\ Arcos de Jalón, 118, 28037, Madrid, Spain \\ ${ }^{3}$ Optics Department, Physics Faculty, Universidad Complutense de Madrid, \\ Ciudad Universitaria s/n, 28040, Madrid, Spain \\ ${ }^{*}$ Corresponding author: jagomezp@ fis.ucm.es
}

Received 30 July 2010; accepted 11 August 2010;

posted 18 August 2010 (Doc. ID 132558); published 17 September 2010

\begin{abstract}
This paper presents a deflectometric technique to measure the power of an ophthalmic lens as perceived by the user. It is based on a calibrated camera acting as a pinhole in order to measure ray deflection along the same path as the visual axis when the lens is held in front of the eye. We have analyzed numerically the accuracy of our technique, and it has been compared experimentally with a commercial "lens mapper" and with the real user power calculated from the measured topography of the lens surfaces to state the reliability and accuracy of the presented technique. (C) 2010 Optical Society of America
\end{abstract}

OCIS codes: $\quad 120.2650,110.2960,150.6910$.

\section{Introduction}

When an ophthalmic lens user looks through his eyeglasses, we found the classical lens-rotation eye scheme [1] depicted in Fig. 1. A small light bundle coming from the object is refracted by the lens and passes through the eye's pupil (in this model, it is customary to represent the rotating eye by a fixed pupil located at the rotation center of the eye [1]). The small size of the eye's pupil $(4-6 \mathrm{~mm})$ compared to the lens diameter, combined with the small size of the foveal field of view of the human's eye means that all the light beams entering the eye for a given object position go through a small patch of the lens surface (surrounding the point at which the principal ray of the bundle intercepts it). This fact is a key point in the design of ophthalmic lenses, and it explains the working principle of multifocal lenses based on nonsymmetric aspheric surfaces, such as the progressive

0003-6935/10/275125-08\$15.00/0

(C) 2010 Optical Society of America addition ones. In this context, the user power (power perceived by the lens user) is defined as

$$
F_{u}=V_{B}-V_{A}
$$

where $V_{A}$ is the vergence of the beam coming from the object, measured at the lens surface (see Fig. 1) and $V_{B}$ is the vergence of the output beam at the point $B$ located at the so-called vertex sphere (a sphere centered on the eye's center of rotation that passes by the lens back surface vertex). User power is a parameter of paramount importance in ophthalmic lens design because it represents the true power perceived by the user [1] and it allows a precise and accurate description of the effect of the lens over the user vision [1].

The user power of an ophthalmic lens cannot be measured using a focimeter, whether it be an automatic or manual one. In fact, there are important differences between focimeter-measured power and actual user power [1]. 
Most ophthalmic lenses, either aspherical single vision or aspherical progressive addition lenses, are designed by optimizing the curvature distribution of the aspherical surface. The power measured by standard lensmeters depends only on the curvatures of the surfaces and on the lens thickness, as the ray path of light source in these instruments is parallel to the local normal vector of the back surface of the lens at the measuring point. However, when the lens is held in the wearing position, as in Fig. 1, the beam no longer intersects the surfaces along the normal direction, and the power must be computed with Eq. (1). The situation, then, is that the nominal power of the lens matches the lensmeter outcome, but it does not match the power required by the user (user power) [2,3]. During the past few years, new manufacturing techniques have been developed for the ophthalmic industry. In particular, the so-called free-form generators may grind and polish a lens surface with almost arbitrary shape and high accuracy. This technology allows the production of unique aspheric surfaces for each user, taking into account the prescription and the actual position of the lens with respect to the eye. As a result, new lenses are being optimized, trying to provide the right user power at every sight direction. The main problem with these lenses is that their power, as measured by a standard lensmeter, does not match the nominal power (the refractive error of the patient as measured in the prescription room). Lens manufacturers are then providing two different power values: the one that is going to be measured with a lensmeter, and the nominal power, which is supposed to match the user power. The main problem is that currently, there are no practical ways to measure the user power, and the opticians and eye doctors lack convenient ways to assess real lens performance.

Currently, there is a range of instruments able to measure lens power over the whole extension of an ophthalmic lens surface. These "power mappers" provide the optician with a complete picture of the power variation across the lens surface. However, the power measured by these instruments is not the true power experimented by the user-it is roughly

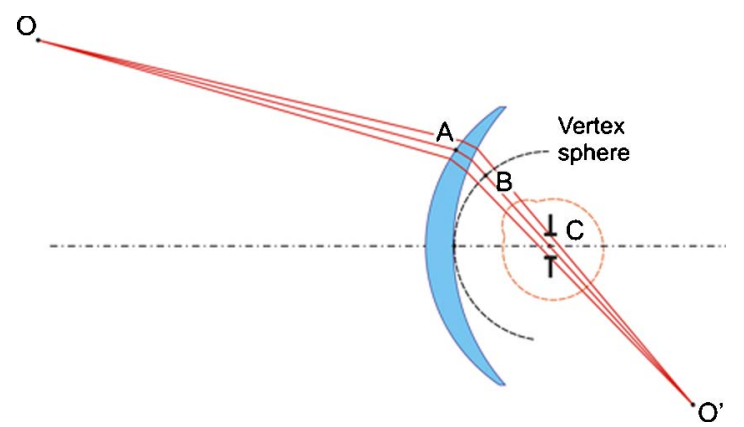

Fig. 1. (Color online) Ophthalmic lens-rotating eye scheme. An object $O$ is observed through an ophthalmic lens by a rotating eye, represented by a diaphragm located at the rotation center of the eye, point $\mathrm{C}$. The vertex sphere is also represented in this picture. equivalent to the focimeter power [4-6]. There are also some proposals for measuring the user power of a lens with a standard lensmeter by rotating the lens above the support of the lensmeter and scanning through all the possible angular directions [3]. Although this procedure is theoretically valid, it requires an impractical amount of time (more than an hour for a medium density scan).

In this paper, a technique for measuring the user power of an ophthalmic lens based on moiré deflectometry is proposed. It is based on the simple deflectometric technique proposed by Massig [7], using a calibrated camera acting as a pinhole with the projection center of the camera precisely located at the point, relative to the lens, at which the rotation center of the eye should be. In this way, it is possible to measure the ray deflections in the same operating conditions as the user's eye, and an approximation of the user power can be derived from them, so a map of the user power of the lens is obtained.

The paper is organized as follows. In the next section, the theoretical basis of our method is given. Afterward, we discuss the accuracy of our technique and perform an empirical and numerical comparison between our technique and a commercial lens mapper based on classical deflectometry. Also, the true user power is obtained for the tested lens and compared with our results. Finally, conclusions are drawn to finish the paper.

\section{Measurement of Prismatic Power Perceived by the Lens User}

The pinhole model is a simple way to represent the image forming properties of a camera by means of a projective transform between two coordinate systems [요미. In Fig. $\underline{2}$, we have represented the

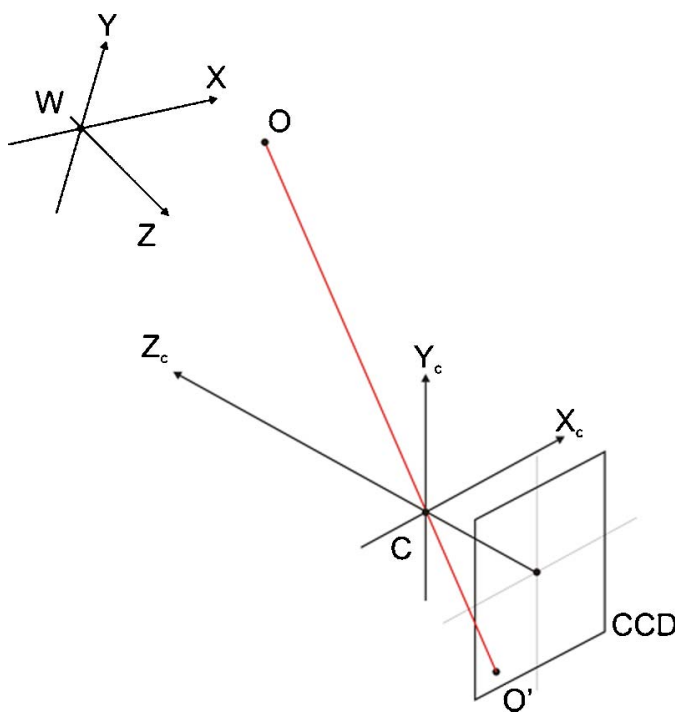

Fig. 2. (Color online) Image formation by a pinhole camera. Object point $O$ is mapped onto an image point $O^{\prime}$, defined by the intersection of the principal ray (which passes through the projection center C) with the CCD plane. Note that both the World Reference System and the Camera Reference System have been represented in this figure as $\mathrm{W}$ and $\mathrm{C}$ for its respective origins. 
two coordinate systems involved in a projective transformation, the World Coordinate System (WCS) and the Camera Coordinate System (CCS). Using homogeneous coordinates [8], if the coordinates of a given point (object point) in the WCS are $M=$ $(X, Y, Z, 1)^{T}$, the coordinates of the image point (measured in the CCS) are $m=(u, v, 1)^{T}$, with $T$ representing the transpose operator. According to Zisserman [8], if the pure projective camera approximation (ignoring the effects of image distortion) holds, the coordinates of image and object point are related through the following expression:

$$
\begin{aligned}
\left(\begin{array}{l}
u \\
v \\
1
\end{array}\right) & =\left[\begin{array}{cccc}
f_{x} & 0 & c_{x} & 0 \\
0 & f_{y} & c_{y} & 0 \\
0 & 0 & 0 & 1
\end{array}\right]\left[\begin{array}{cccc}
r_{X X} & r_{X Y} & r_{X Z} & t_{X} \\
r_{Y X} & r_{Y Y} & r_{Z Z} & t_{Y} \\
r_{Z X} & r_{Z Y} & r_{Z Z} & t_{Z} \\
0 & 0 & 0 & 1
\end{array}\right]\left(\begin{array}{c}
X \\
Y \\
Z \\
1
\end{array}\right) \\
& =\mathbf{P} \cdot\left(\begin{array}{c}
X \\
Y \\
Z \\
1
\end{array}\right),
\end{aligned}
$$

where the first matrix of the right side of Eq. (2) represents the so-called "intrinsic" [8] camera parameters (focals $f_{X}$ and $f_{Y}$, and principal point coordinates $c_{X}$ and $c_{Y}$ in appropriate units). The second matrix of the right side of Eq. (2) stands for the "extrinsic" parameters given by a $\overline{3} \times 3$ rotation matrix $R$, whose components are the $r_{i j}$ coefficients of Eq. (1) and a three-dimensional vector, $\mathbf{t}=\left(\begin{array}{lll}t_{X} & t_{Y} & t_{Z}\end{array}\right)^{T}$. These extrinsic parameters take into account the fact that the orientation and location of the center of the WCS and CCS are different (see Fig. 2). We have denoted by $\mathbf{P}$ the matrix resulting from the product of these matrices, and it represents the action of the projective camera [8] over the object points to get the image ones (forward projection). Both the extrinsic and intrinsic camera parameters can be obtained through a calibration procedure similar to the one described by Zhang [9]. In this paper, we have used the calibration technique employed by the Camera Calibration Toolbox for MATLAB developed by Bouget [10]. As the Camera Calibration Toolbox allows the correction of the lens distortions, we will suppose, without loss of generality, that our calibrated cameras are distortion-free and, consequently, the projective camera approximation holds.

If we have a calibrated camera, we can locate the projection center of the camera exactly at the same distance of the back lens surface of that occupied by the rotation center of the eye. For this purpose, we put three marks (aligned horizontally) on the back lens surface with the known distances between. If these marks are referred in the Camera Coordinate System, Eq. (2) can be rewritten as

$$
\left(\begin{array}{c}
u^{i} \\
v^{i} \\
1
\end{array}\right)=\left[\begin{array}{cccc}
f_{x} & 0 & c_{x} & 0 \\
0 & f_{y} & c_{y} & 0 \\
0 & 0 & 0 & 1
\end{array}\right]\left(\begin{array}{c}
X^{i}{ }^{c} \\
Y^{i}{ }^{c} \\
Z^{i}{ }_{c} \\
1
\end{array}\right),
$$

where $i=[1,2,3]$ labels the different marks, and $M^{i}{ }_{c}=\left(X_{c}^{i}, Y^{i}{ }_{c}, Z^{i}, 1\right)^{T}$ denotes the coordinates of the $i$ th mark, referred in the camera reference frame $[8,11]$. As the distance between mark coordinates is previously known and the distance between marks can be obtained in the camera units, we can get from Eq. (3) an overdetermined system with $Z^{i}{ }_{c}$ being the unknown variables. Moreover, as the lens is placed with its optical axis parallel to that of the CCD camera, we can consider that the value of the $Z_{c}^{i}$ coordinate is approximately the same for all the marks, and we can get its mean value $Z_{c}$. Once we have obtained the distance $Z_{c}$ between the camera projective center and the back lens surface, it is possible to move the lens closer or farther away with respect to the camera in order to place it at user conditions.

The accurate location of the lens with respect to the projection center of the CCD camera allows us to measure the prismatic power in conditions close to the real ones with a deflectometric technique based on Massig's [7]. In Fig. 3, the experimental setup employed is depicted. It consists of a flat screen and a camera (characterized, in the pinhole model, by the CCD plane and the projection center) connected to a computer, and a lens holder placed between them in such a way that the distance between the back vertex of the lens and the projection center of the camera is about $27 \mathrm{~mm}$, so the projection center of the camera lays exactly at the same location of the rotation center of the eye.

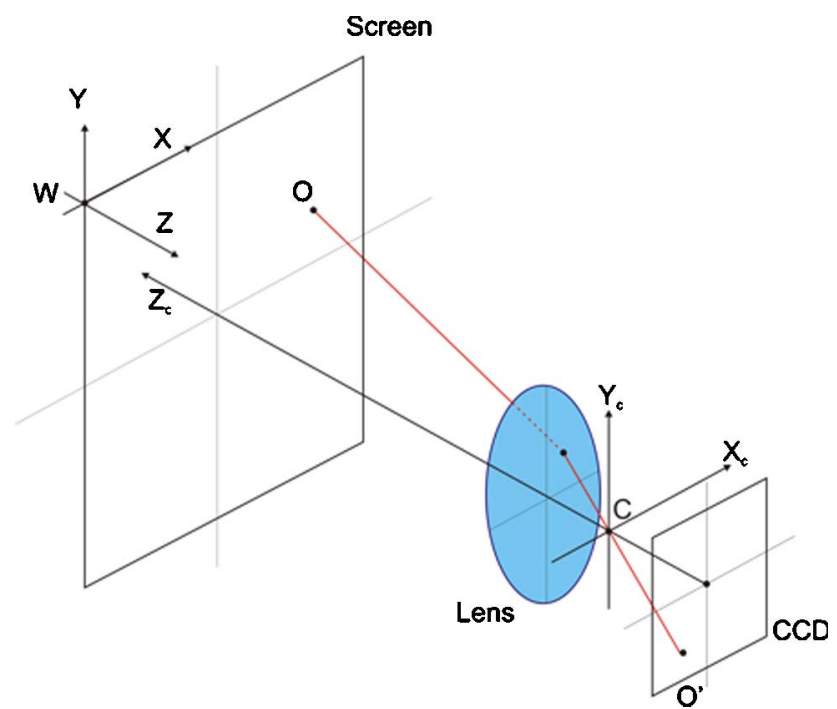

Fig. 3. (Color online) Scheme of the experimental setup employed to measure the prismatic power formed by a flat screen, a CCD camera (modeled as a pinhole camera), and the lens to be measured. The World and Camera Reference Systems employed are also depicted with points $\mathrm{W}$ and $\mathrm{C}$ as the centers of these reference systems, respectively. 
In order to apply our technique, it is necessary to distinguish accurately between different object points. This is achieved using a mixed gray-coding and phase-shifting technique [11], so every object point will be matched with two absolute phase values $\left[\Phi_{X}, \Phi_{Y}\right]$, which are linearly related to WCS coordinates $X$ and $Y$ of the object point (by definition, $Z=$ 0 for every object point) as

$$
\Phi_{X}=\frac{2 \pi}{p_{X}} X, \quad \Phi_{Y}=\frac{2 \pi}{p_{Y}} Y,
$$

where $p_{X}$ and $p_{Y}$ are the horizontal and vertical periods, respectively, of the sinusoidal fringe pattern used in the phase-shifting technique [11].

The selected technique [11] for object labeling requires the projection of 12 images (eight gray-coded and four phase-shifted sinusoidal) in the screen for each direction. The CCD camera captures these images, and from them, we get two phase maps (in camera coordinates), $\Phi_{X}(u, v)$ and $\Phi_{Y}(u, v)$. As the phase maps referring to the WCS, $\left[\Phi_{X}, \Phi_{Y}\right]$ are linearly related to the WCS coordinates $X$ and $Y$, it is possible to use Eq. (4) to get the location of an object point in the LCD screen ( $[X, Y]$ coordinates) from the measured CCS phase maps $\Phi_{X}(u, v)$ and $\Phi_{Y}(u, v)$, with $Z$ being coordinate zero for all the object points [11].

In order to calculate the ray deviation, we have represented in Fig. 4 the trajectory of a principal light ray (contained in the plane defined in the CCS by the $Y_{c}$ and $Z_{c}$ axes), which passes through the aperture diaphragm of the optical system formed by the ophthalmic lens and the pinhole camera (the pinhole acts as the aperture diaphragm of the whole system). The ray comes from the object point $O$, passes through the lens by the point $P$, and impinges on

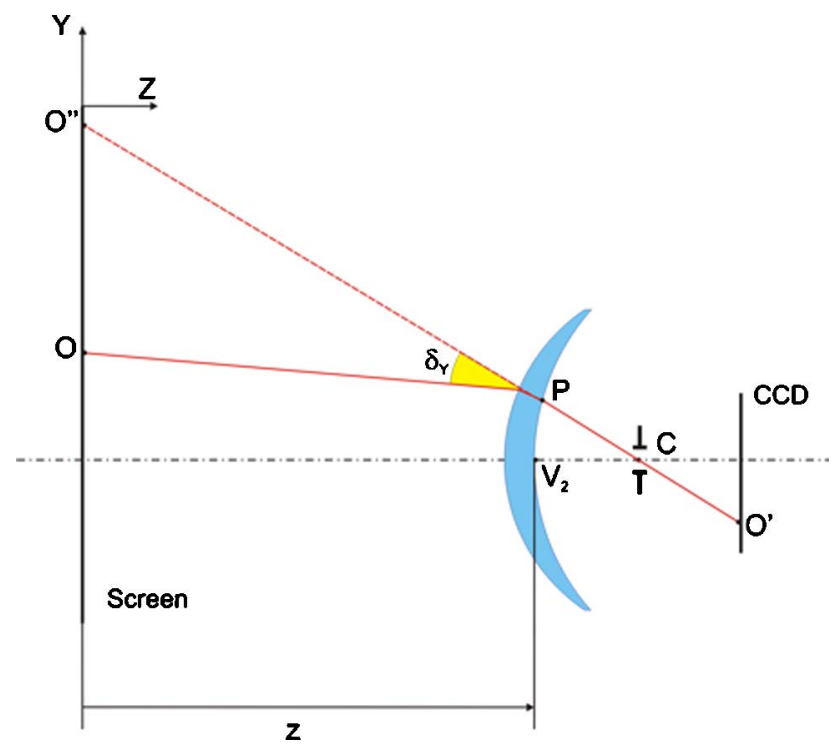

Fig. 4. (Color online) Image formation from an ophthalmic lens, plus a pinhole camera. Object point $\mathrm{O}$ is imaged onto the image plane at $O^{\prime}$, where the principal ray intercepts the CCD plane. The backprojection of $O^{\prime}$ gives the point $\mathrm{O}^{\prime \prime}$ at the screen. the image point $O^{\prime}$ located at the CCD plane. This image point is backprojected to the point $O^{\prime \prime}$ located at the object plane. The component $\delta_{Y}$ of the angular deviation (prismatic effect) can be obtained from the triangle formed by the points $O, P$, and $O^{\prime \prime}$ (see Fig. $\underline{4}$ ) using the following expression:

$$
\delta_{Y}=\tan \left(\frac{Y_{O^{\prime \prime}}-Y_{O}}{z}\right)
$$

where $Y_{O}$ and $Y_{O^{\prime \prime}}$ are the vertical coordinates of the position vectors of $O$ and $O^{\prime \prime}$, respectively, and $z$ is the horizontal distance between the object plane and the vertex of the back surface of the lens (see Fig. 4). Obviously, Eq. (5) is an approximation of the true prismatic effect at point $P$, but it represents a good estimation of the prismatic power perceived by the lens user. For a general skew ray (not contained in the $Y_{c} Z_{c}$ plane), the prismatic effect components are given by the following equations:

$$
\delta_{X}=\tan \left(\frac{X_{O^{\prime \prime}}-X_{O}}{z}\right), \quad \delta_{Y}=\tan \left(\frac{Y_{O^{\prime \prime}}-Y_{O}}{z}\right)
$$

To get the coordinates $\left[X_{O}, Y_{O}\right]$ and $\left[X_{O^{\prime \prime}}, Y_{O^{\prime \prime}}\right]$, it is necessary to take two measurements. First, a measurement is taken without the lens, so for each pixel of the CCD camera, we get the coordinates $\left[X_{O^{\prime \prime}}, Y_{O^{\prime \prime}}\right]$ from the phase values $\Phi_{X}(u, v)$ and $\Phi_{Y}(u, v)$ obtained at this point, using Eq. (4). Note that, according to Fig. 4, point $O^{\prime \prime}$ corresponds to the backprojection of the image point $O^{\prime}$. Therefore, when the ophthalmic lens is removed, the CCD camera forms the image of $O^{\prime \prime}$ at the point $O^{\prime}$, and the phase values $\Phi_{X}(u, v)$ and $\Phi_{Y}(u, v)$ corresponding to the pixel $(u, v)$ of the camera are related to coordinates $\left[X_{O^{\prime \prime}}, Y_{O^{\prime \prime}}\right]$ through Eq. (4). Afterward, we take a measurement with the lens, and the coordinates $\left[X_{O}, Y_{O}\right]$ are obtained using the same procedure (because, in this case, the system lens CCD camera forms the image of point $O$ at point $O^{\prime}$ and the phase values measured at $O^{\prime}$ correspond to the coordinates of $O$ ). To calculate the prismatic power, it is necessary to know the distance $z$ between the screen and the lens. This distance is obtained from the calibration data of the CCD camera, because from these data, the location of the screen referred to the CCS is extracted and remains only to subtract the distance to the center of rotation obtained previously (in our experiment, $27 \mathrm{~mm}$ ) in order to get $z$. Once we get the prismatic power by Eq. (5), it is easy to calculate an approximation of the user power by means of the local dioptric power matrix $[12,13]$ through the derivatives of deflections $\delta_{X}$ and $\delta_{Y}$. In the next section, we will test the accuracy of the proposed technique both numerically and empirically. 


\section{Experimental Results}

In order to check the accuracy of the proposed technique, we have performed first a numerical simulation using Zemax, a commercial ray-tracing software. We have simulated the optical system formed by the ophthalmic lens (a monofocal one with negative power) and the CCD objective (modeled as a paraxial system formed by two thin lenses with the same cardinal points as the real CCD objective employed in the experiment) in three configurations: configuration $\mathrm{A}$, with the entrance pupil of the camera placed $27 \mathrm{~mm}$ behind the lens; configuration $\mathrm{B}$, with the projection center of the objective placed $27 \mathrm{~mm}$ behind the lens; and configuration $\mathrm{C}$, with the entrance pupil of the camera placed $60 \mathrm{~mm}$ behind the lens [we could not further separate the CCD from the lens without excessively limiting the field of view-see Fig. 5(c)]. These configurations simulate: the real prismatic effect in user's condition (A), the proposed technique (B), and Massig's technique (C). For the three configurations, the distance between screen and lens is the same $(300 \mathrm{~mm})$ in order to show the effect of the relative location of the ophthalmic lens and CCD in the measurement of ray deflection. In Fig. 5 , we can see the three config-

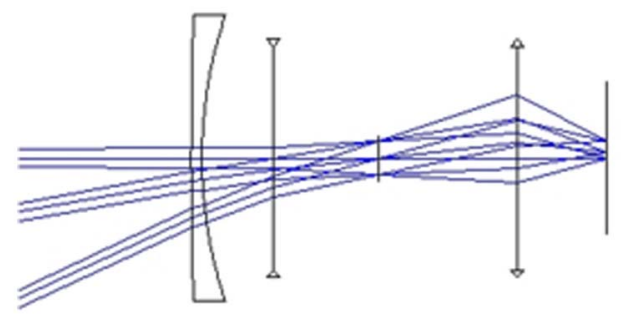

(a)

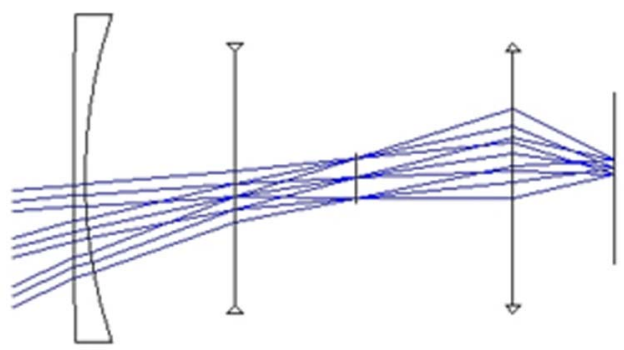

(b)

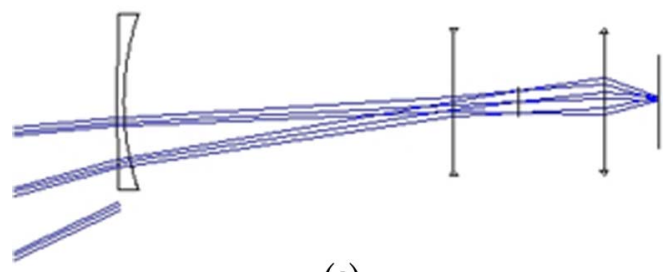

(c)

Fig. 5. (Color online) Ray-tracing drawings of the three configurations studied: (a) configuration $\mathrm{A}$, with the objective entrance pupil placed at the eye's rotation center, (b) configuration $\mathrm{B}$, with the objective nodal point (space object) placed at the same point, and (c) configuration $\mathrm{C}$ with the objective separated a greater distance from the lens. urations proposed and the ray-tracing through the lens CCD objective system. The CCD objective has been simulated as a paraxial system for two reasons: 1) in the pinhole model, the only relevant aberration is distortion, but, as mentioned before, we can correct distortion, so we may suppose, without loss of generality, that our objective is a paraxial one; and 2) for a paraxial objective, the projection center could be identified with the object nodal point [14], so we can accurately locate this point $27 \mathrm{~mm}$ behind the lens.

In Table 1 , the ray deviation calculated for a set of object points contained in the $Y_{c} Z_{c}$ plane, defined by the field angles $0,5^{\circ}, 10^{\circ}, 15^{\circ}, 20^{\circ}$, and $25^{\circ}$ is presented. It should be noticed that, for the configuration $\mathrm{A}$, the ray deviation has been calculated as the difference between the angles subtended by the principal ray and the optical axis at the front and back surface of the lens, respectively. To calculate the ray deviation in the configurations $\mathrm{B}$ and $\mathrm{C}$, we have used Eq. (6), so we have determined the prismatic power from the position of the object and image points (calculated by the ray-tracing program).

As it can be seen in Table 1 , the prismatic power calculated using our technique is a better approximation of the actual prismatic power experienced by a lens user than that calculated using Massig's technique for high field angles, while in the paraxial zone, the differences are about the same. Therefore, it has been demonstrated that the relative distance between the lens and the CCD objective has influence on the calculation of the prismatic effect, and it should be taken into account in order to get a better approximation of the lens user power.

The experimental results obtained are presented in Figs. 6(a) and 6(b), where we can see the prismatic power maps obtained with our system for the central zone of a standard progressive addition lens (about $\pm 25^{\circ}$ around the fitting cross, which marks the point to be aligned with the center of the pupil in a standard fitting). We have employed a 15 in. LCD monitor and a $2 / 3$ in. CCD camera with a wide-field objective (focal length $4.8 \mathrm{~mm}$ ) with the distance between monitor and lens fixed at $300 \mathrm{~mm}$. The field of view of the system is enough for the measurement of a cut spectacle lens for the standard frames used currently, and it could be improved by changing

$\begin{gathered}\text { Table 1. Prismatic Power (Ray Deviation) Both in Degrees and } \\
\text { Prismatic Diopters Calculated in Three Different Experimental } \\
\text { Configurations for the Lens-Camera System }{ }^{a}\end{gathered}$
\begin{tabular}{rcccccc}
\multicolumn{7}{c}{} \\
\hline$\omega_{O}\left({ }^{\circ}\right)$ & $\delta_{\text {real }}(\Delta)$ & $\delta_{\text {ap }}(\Delta)$ & $\delta_{\text {Mas }}(\Delta)$ & $\delta_{\text {real }}\left({ }^{\circ}\right)$ & $\delta_{\text {ap }}\left({ }^{\circ}\right)$ & $\delta_{\text {Mas }}\left(^{\circ}\right)$ \\
\hline 5 & 1.15 & 0.77 & 1.43 & 0.66 & 0.44 & 0.82 \\
10 & 2.32 & 1.47 & 2.95 & 1.33 & 0.84 & 1.69 \\
15 & 3.57 & 2.48 & 4.71 & 2.04 & 1.42 & 2.70 \\
20 & 4.90 & 3.86 & 6.83 & 2.81 & 2.21 & 3.92 \\
25 & 6.36 & 5.43 & - & 3.64 & 3.11 & - \\
\hline
\end{tabular}

${ }^{a} \omega_{O}$ is the field angle subtended by the object, $\delta_{\text {real }}$ is the actual ray deviation perceived by the user, $\delta_{\text {ap }}$ is the prismatic power estimated by the proposed technique, and $\delta_{\text {Mas }}$ is the ray deviation estimated by the Massig's technique. 
Prismatic power $\delta_{x}(\Delta)$

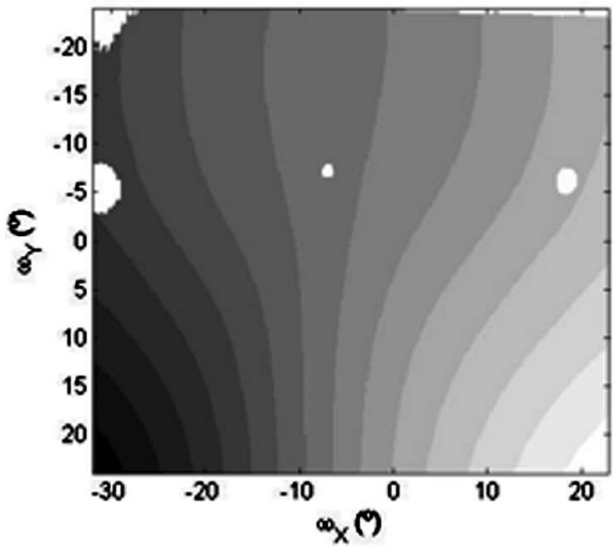

(a)

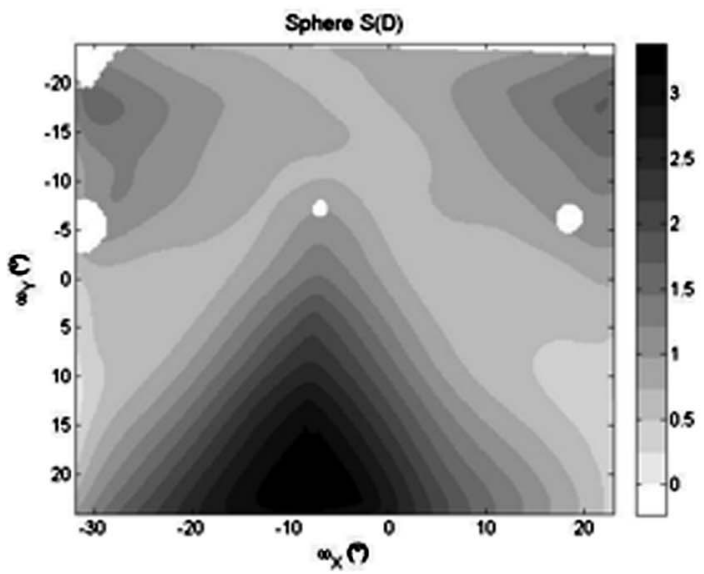

(c)

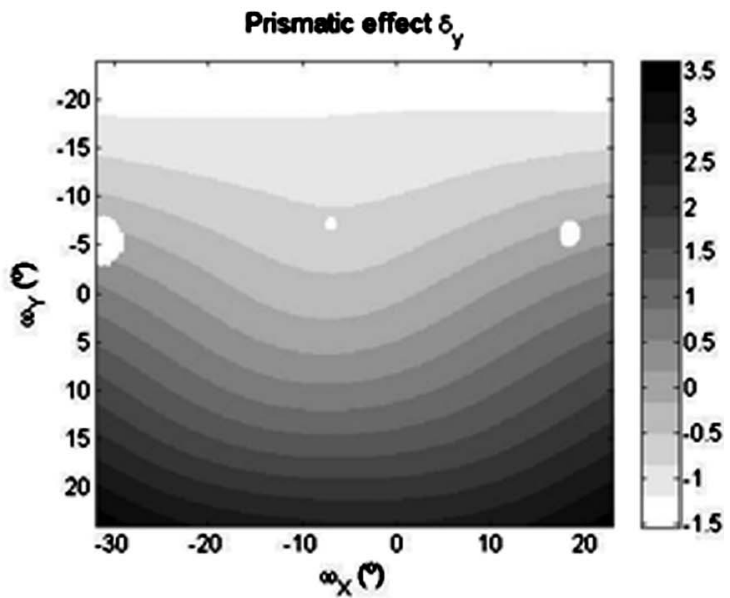

(b)

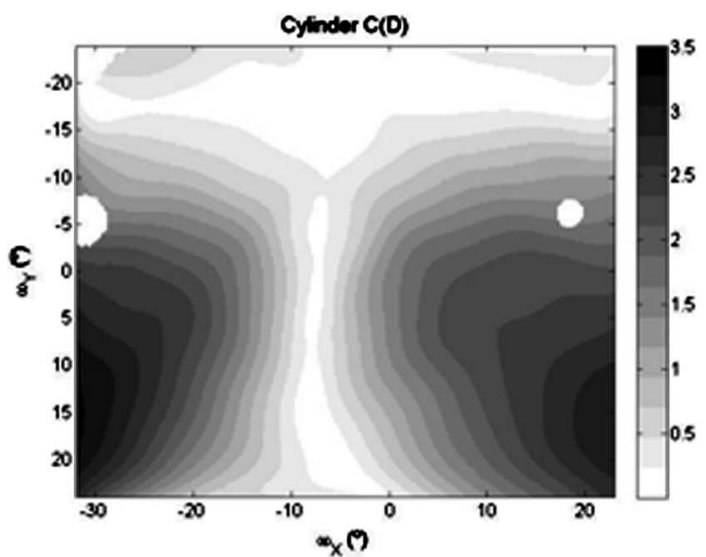

(d)

Fig. 6. Prismatic power obtained in the (a) horizontal and (b) vertical directions using our technique for a progressive addition lens. Also represented are the (c) spherical and (d) cylindrical power maps obtained from them.

the distance between lens and screen and/or using a bigger LCD monitor. Measurement time, including all the necessary computations, is slow compared with commercial lens mappers (about $5 \times \min$ for the lens), but it could be greatly reduced by some improvements, such as triggered image adquisition, compiled software, fewer measured points, and so on. The corresponding sphere and cylinder power maps are depicted in Figs. 6(c) and 6(d), respectively. In these plots, the typical features of a progressive addition lens can be observed: the sphere power progression down the lens [Fig. 6(c)], and the

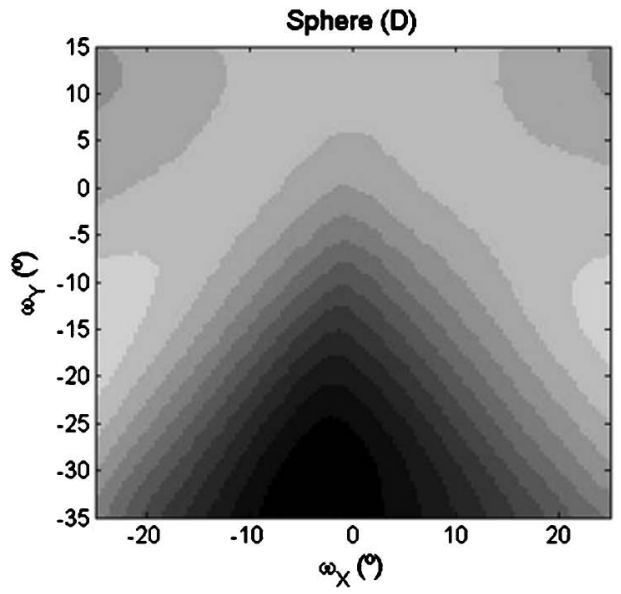

(a)
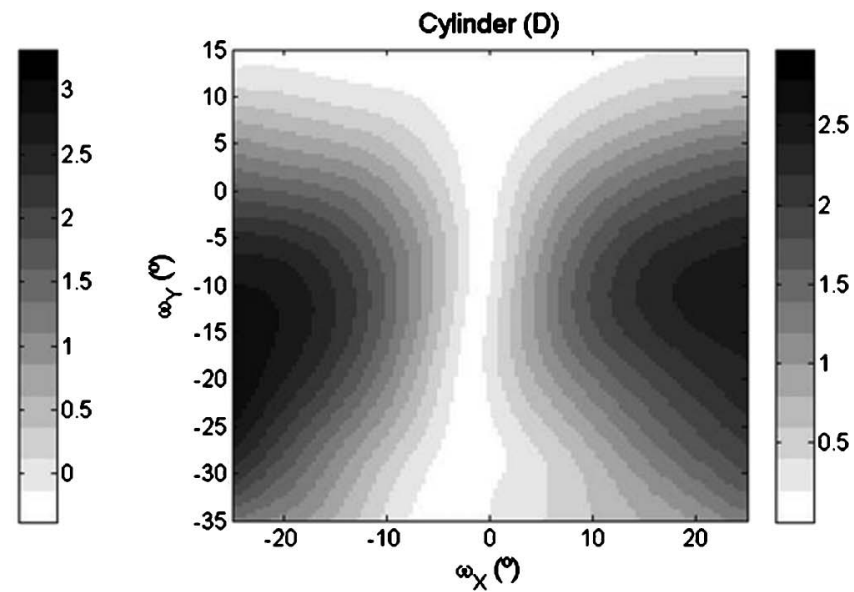

(b)

Fig. 7. (a) Spherical and (b) cylindrical power maps of the same lens from Fig. $\underline{6}$, obtained using a lens mapper (Rotlex's Class Plus). 


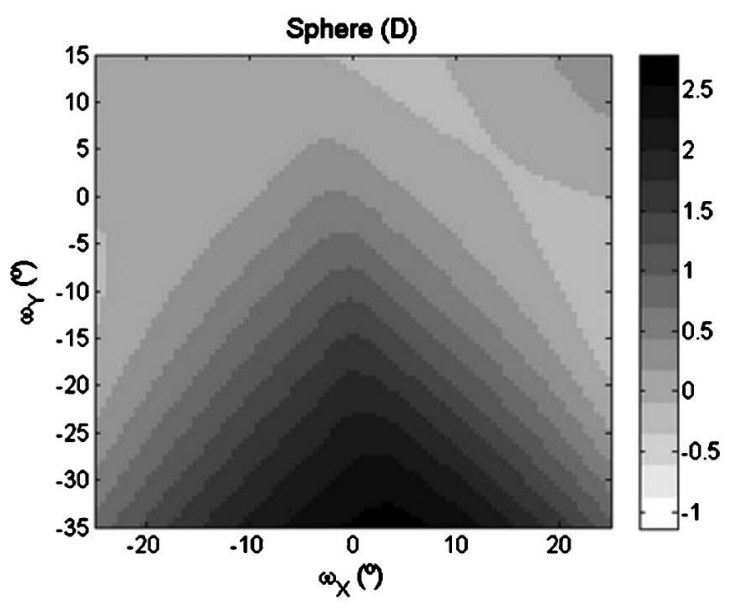

(a)

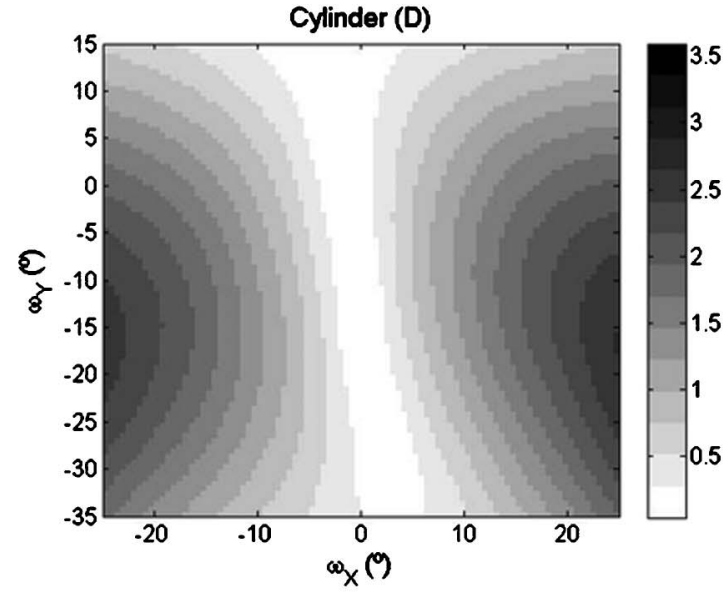

(b)

Fig. 8. Actual (a) spherical and (b) cylindrical user power maps derived through ray tracing from the measured surface of the progressive addition lens studied in this paper.

progressive corridor [Fig. 6(d)] formed by the distribution of cylindrical power by both sides of the umbilical line [1].

In order to test the results obtained, we have first measured the spherical and cylindrical power maps of the test lens using a commercial lens mapper (Class Plus from Rotlex [15]). These maps are shown in Figs. 7(a) and 7(b), respectively. There is a good quantitative agreement between the two spherical power maps, while there are slight differences between the cylindrical power maps. As it is shown in Fig. 6(d), the cylinder map obtained with the proposed methods presents higher peak values than those measured using the lens mapper. Moreover, the shape of both maps is slightly different, as well. This is something to be expected, as the cylinder perceived by the user in the two lobes at both sides of the corridor is typically bigger than the values measured by lensmeters or standard lens mappers [1].

Finally, we have measured the lens surfaces with a mechanical profiler that directly measures sag values with an accuracy of $\pm 0.2 \mu \mathrm{m}$, and we have computed the true spherical and cylindrical power as perceived by the lens user by means of exact ray tracing. These maps are depicted in Figs. 8(a) and $8(\mathrm{~b})$. Again, we find a good agreement between the power maps obtained with our method (note that, in this case, the peak values and the shape of the calculated cylindrical user power map are closer to those obtained with our technique). Therefore, we can conclude that with our technique, we can get a good estimation of the spherical and cylindrical power that is actually perceived by the lens user.

\section{Conclusions}

It is presented in this paper a deflectometric technique to measure the ray deflection (prismatic power) for an ophthalmic lens in conditions close to those when the lens is actually used. In this way, we get a good estimation of the prismatic power experimented by a lens wearer when looking through his eye- glasses. The presented technique requires a simple experimental setup (composed by an LCD screen and a CCD camera), and it relies on the properties of a calibrated camera to locate accurately the projection center of the camera in the same location as that occupied by the rotation center of the eye when the lens is actually used. In this way, we can assure that the prismatic effect is measured in near-use conditions.

In order to establish the difference between our technique and similar deflectometric methods, we have performed a simulation using a ray-tracing program showing the dependence of the ray deflection on the distance between lens and CCD camera, and the importance of locating the projection center of the camera in the exact location of the rotation center of the eye in order to get the actual power perceived by the user. We have also performed two experiments comparing our method with a similar deflectometric technique (a commercial lens mapper, in this case) and calculating the actual user power of the measured lens. We have concluded from these experiments that our technique provides us with a fair estimation of the actual power perceived by the lens user in true usage conditions.

This work has been financially supported by the Spanish Ministry of Science and Technology through the project number DPI2009-09023. It has also benefited from the framework of the Spanish Optometry Network.

\section{References}

1. B. Bourdoncle, J. P. Chauveau, and J. L. Mercier, "Traps in displaying optical performances of a progressive-addition lens," Appl. Opt. 31, 3586-3593 (1992).

2. D. A. Atchinson, M. Kris, J. E. Sheedy, and I. L. Bailey, "Use of the Humphrey Lens Analyzer for off-axis measurements of spectacle lenses," Optom. Vis. Sci. 68, 299-308 (1991).

3. D. A. Atchinson and M. Kris, "Off- axis measurement of a plano distance power progressive addition lens," Ophthal. Physiol. Opt. 13, 322-326 (1993). 
4. K. Gnanvo, Z. Y. Wu, J. L. de Bougrenet de la Tocnaye, and L. Liu, "Large-aperture automatic focimeter for the measureement of optical power and other optical characteristics of ophthalmic lenses," Appl. Opt. 41, 5997-6005 (2002).

5. H. Canabal and J. Alonso, "Automatic wavefront measurement technique using a computer display and a chargecoupled device camera," Opt. Eng. 41, 822 (2002).

6. J. A. Quiroga, D. Crespo, and E. Bernabeu, "Fourier transform method for automatic processing of moiré deflectograms," Opt. Eng. 38, 974-982 (1999).

7. J. H. Massig, "Measurement of phase objects by simple means," Appl. Opt. 38, 4103-4105 (1999).

8. R. Hartley and A. Zisserman, Multiple View Geometry in Computer Vision, 2nd ed. (Cambridge University Press, 2008).

9. Z. Zhang, "A flexible new technique for camera calibration," IEEE Trans. Pattern Anal. Mach. Intell. 22, 1330-1334 (2000).
10. J. Y. Bouget, “Camera calibration toolbox for MatLab," http:// www.vision.caltech.edu/bouguetj/calib_doc/ (2010).

11. J. Vargas, J. A. Quiroga, and M. J. Terrón, "Flexible calibration procedure for fringe projection profilometry," Opt. Eng. 46, 023601 (2007).

12. J. Alonso, J. A. Gómez-Pedrero, and E. Bernabeu, "Local dioptric power matrix in a progressive addition lens," Ophthal. Physiol. Opt. 17, 522-529 (1997).

13. J. A. Gómez-Pedrero, J. Alonso, H. Canabal, and E. Bernabeu, "A generalization of Prentice's law for lenses with arbitrary refracting surfaces," Ophthal. Physiol. Opt. 18, 514-520 (1998).

14. M. Born and E. Wolf, Principles of Optics, 6th ed. (Pergamon, 1980).

15. O. Kafri, "Noncoherent method for mapping phase objects," Opt. Lett. 5, 555-557 (1980) 